\title{
Crystal structure of primosome loader protein DnaB from Geobacillius stearothermophilus and its interplay with PriA protein for DNA replication restart
}

\author{
Chwan-Deng Hsiao \\ Institute of Molecular Biology, Academia Sinica, Taipei, 115, Taiwan
}

The Gram-positive bacterial DnaB primosomal protein plays a key role in DNA replication and restart as a loader protein for the recruitment of replisome cascade proteins. The DnaB protein is composed of three structural domains: an N-terminal domain, a middle domain and a C-terminal domain. Here, we report the crystal structures of primosome loader protein DnaB encompassing the N-terminal and middle domains from Geobacillius stearothermophilus $\left(G s t \mathrm{DnaB}_{1-300}\right)$. The structure reveals a unique tetrameric organization with two basket-like architectures. To our surprise, the overall tetrameric conformation of GstDnaB ${ }_{1-300}$ in our crystal structure differs significantly from those of previously observed low-resolution structures of DnaB from Bacillus subtilis $(B s u$ DnaB) reported by atomic force microscopy (AFM) and electron microscopy (EM) studies. The tetramer conformation from AFM and EM studies showed that BsuDnaB forms square-like or helical structures, respectively. In addition, from our GST pull-down assays and analytical ultracentrifugation (AUC) suggest that GstDnaB physically interacts with GstPriA from N-terminal and middle domains but not $\mathrm{C}$-terminal domain. This finding is not observed in previous studies. Moreover, from our electrophoresis mobility shift assays (EMSA) studies reveals that GstDnaB binds to ssDNA substrate through its C-terminal domain. These findings highlight the functional difference between the N-terminal plus middle domain and the C-terminal domain, respectively, in terms of PriA binding and ssDNA binding. To further understand the full-length structure of GstDnaB, we also perform the small-angle X-ray scattering (SAXS) analysis. The SAXS result clearly indicates that the overall envelope for full-length GstDnaB was elongated in "X-like" shape, with additional density at the four corners that could adequately accommodate the C-terminal domain of GstDnaB. Taken together, our findings provide insight into the functional role of DnaB primosomal protein in relation to Gram-positive bacterial DNA replication. 